\title{
Informations XXIlème Congrès de la SALF
}

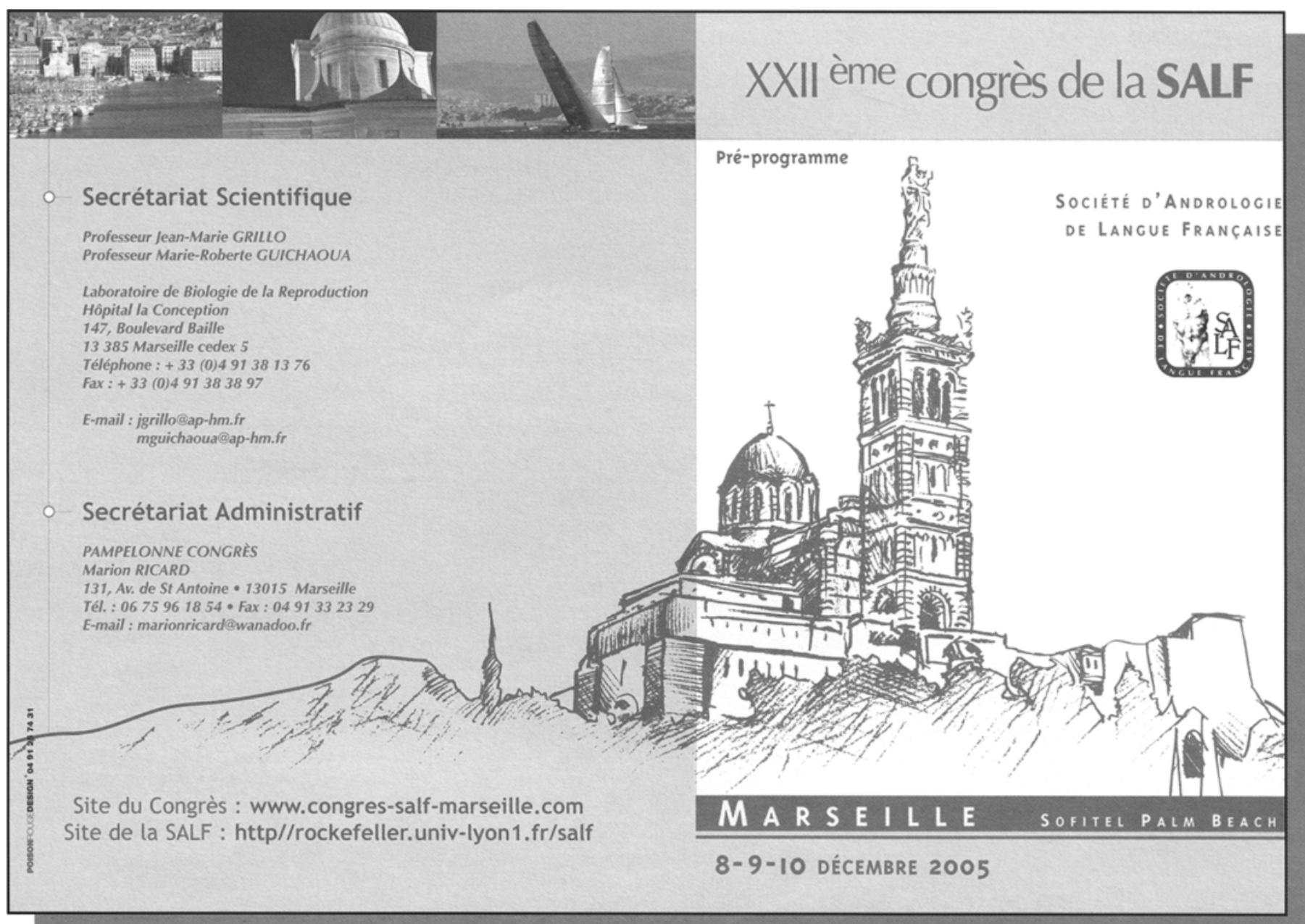




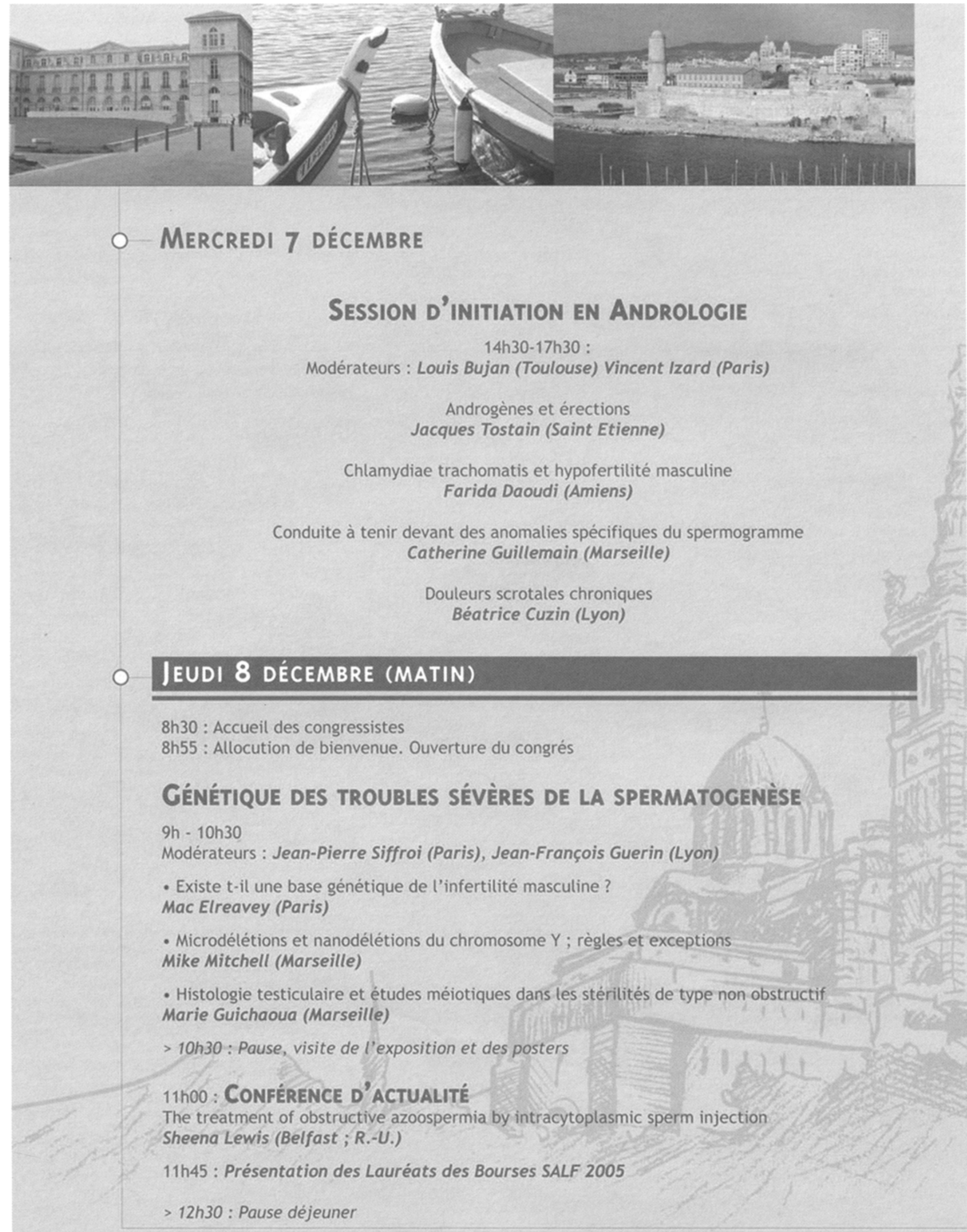




\section{JEUDI 8 DÉCEMBRE (APRÈS-MIDI)}

\section{Chirurgie de la verge}

$14 \mathrm{~h}-16 \mathrm{~h}$

Modérateurs : Pierre Bondil (Chambéry), Jacques Tostain (Saint Etienne)

- Traumatismes de la verge et des organes génitaux externes

Gérard Serment (Marseille)

- Conduite à tenir devant un priapisme

Dominique Rossi (Marseille)

- Résultat de la chirurgie prothétique pour dysérection

Daniel Chevallier (Nice)

- Chirurgie plastique et pathologie de la verge

Dominique Casanova (Marseille)

- Retentissement psychologique de la chirurgie de la verge Béatrice Cuzin (Lyon)

$>16 \mathrm{~h}$ : Pause, visite de l'exposition et des posters

\section{ETHIQUE EN ANDROLOGIE}

$16 \mathrm{~h} 30-18 \mathrm{~h} 30$

Modérateurs : Agnès Noizet (Marseille), Pierre Tourame (Marseille)

- Problèmes éthiques liés à l'autoconservation des spermatozoïdes

- Autoconservation et âges limites Jean-Marc Rigot (Lille)

- Autoconservation et pronostic vital Pierre Jouannet (Paris)

- Ethique en chirurgie andrologique

Alain Jardin (Paris)

- La Sociologie de la paternité est-elle modifiée par les progrès de l'andrologie et de l'AMP Irène Thery (Paris)

18h30: Assemblée Générale de la SALF
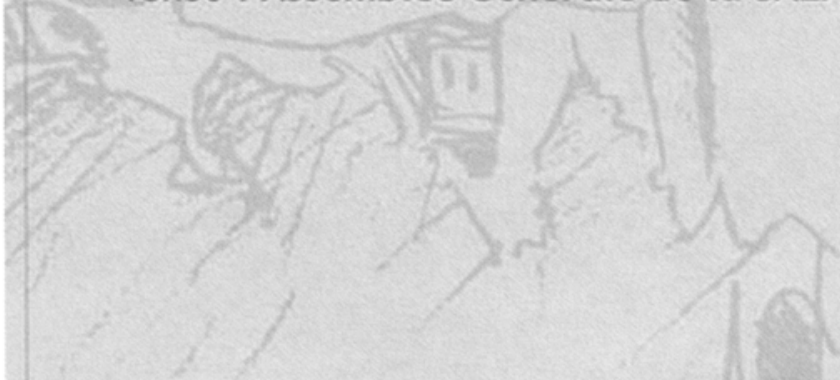


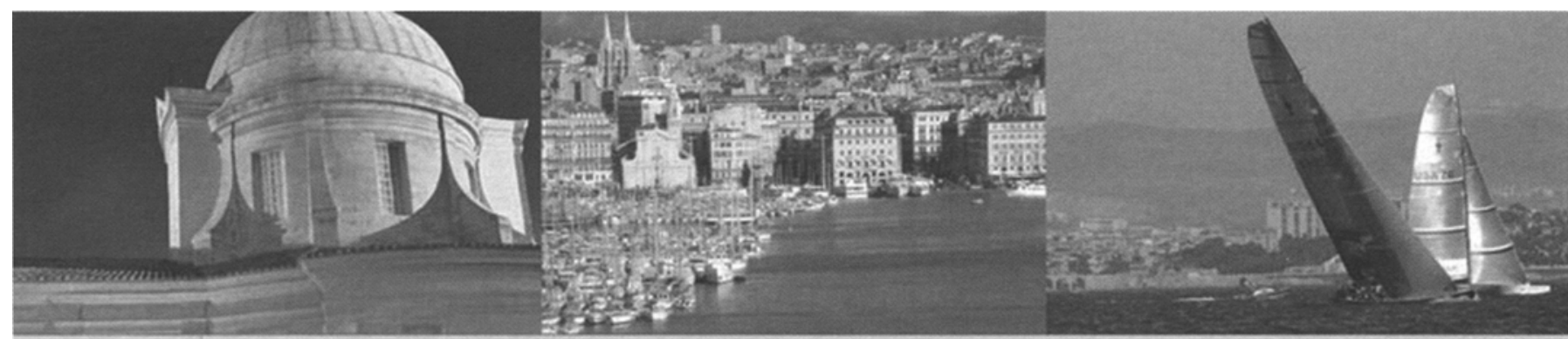

\section{VENDREDI 9 DÉCEMBRE (MATIN)}

\section{Fertilité du blessé médullaire}

$8 \mathrm{~h} 30-10 \mathrm{~h} 15$

Modérateurs : Roger Mieusset (Toulouse), Jean-Marc Soler (Perpignan)

- Conséquences du traumatisme médullaire sur l'érection

Brigitte Schurch (Zurich)

- Conséquence du traumatisme médullaire sur l'éjaculation

François Giuliano (Paris)

- Qualité du sperme et techniques de recueil du sperme chez le blessé médullaire Gilles Karsenty (Marseille)

- Résultats des techniques d'AMP chez le blessé médullaire Laurent Wagner (Nîmes)

$>10 \mathrm{~h} 15$ : Pause, visite de l'exposition et des posters

MAturation des CEllules germinales in Vitro, Quel aVENiR ? $10 \mathrm{~h} 45-12 \mathrm{~h}$

Modérateurs : Philippe Durand (Lyon), Roger Roulier (Marseille)

- Développement du testicule fœtal et néonatal René Habert (Paris)

- Les cultures de cellules germinales pour AMP : état actuel, enseignement des modèles animaux Hervé Lejeune (Lyon)

- Application des tests de génotoxicité aux cellules germinales en coculture Jeanne Perrin (Marseille)

$12 \mathrm{~h}$ : Prix DEA-DESS/SALF

$>12 \mathrm{~h} 30$ : Pause déjeuner

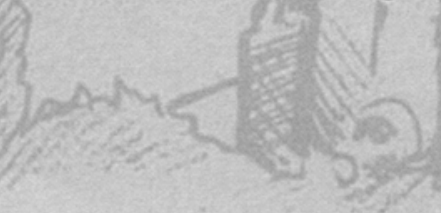




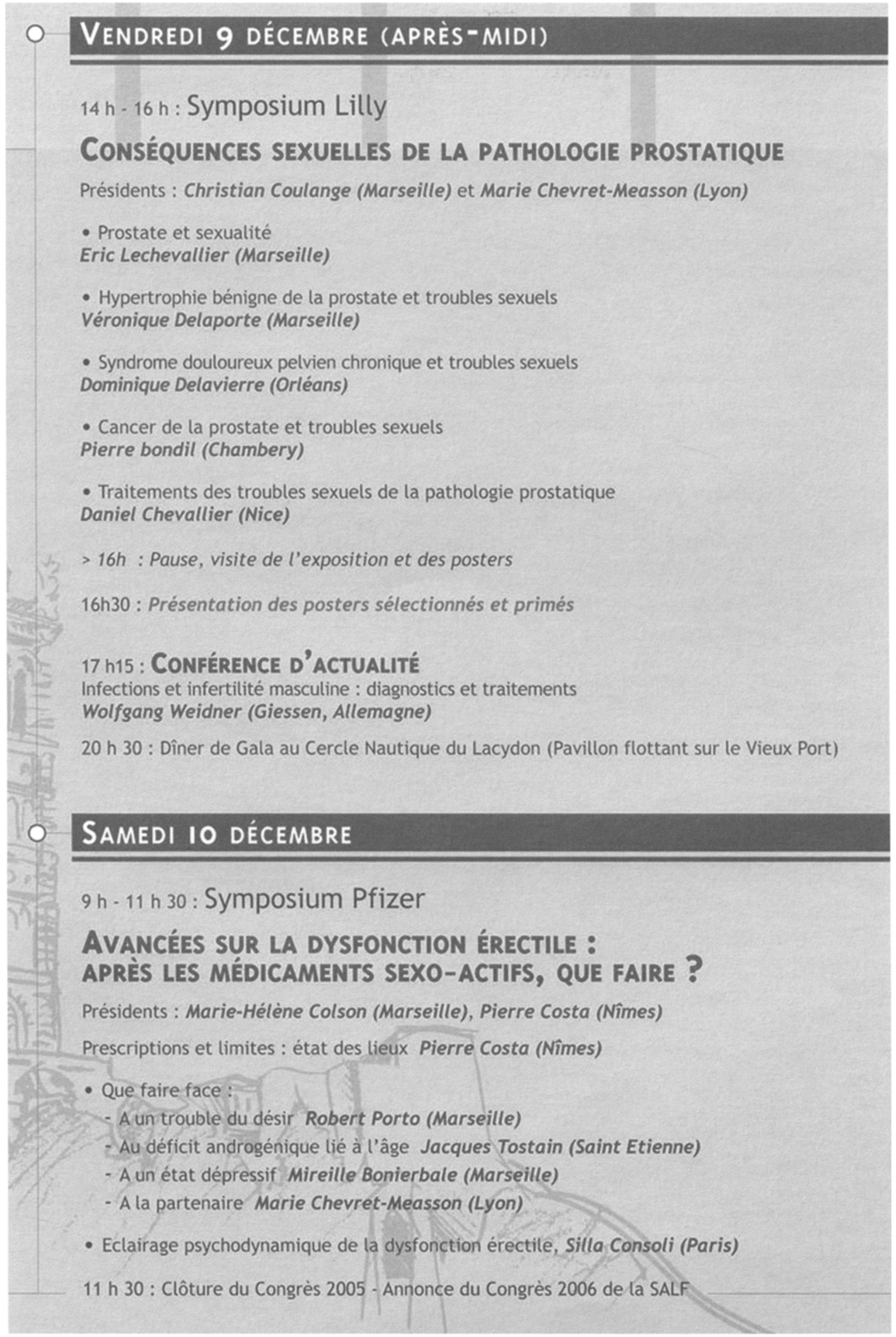




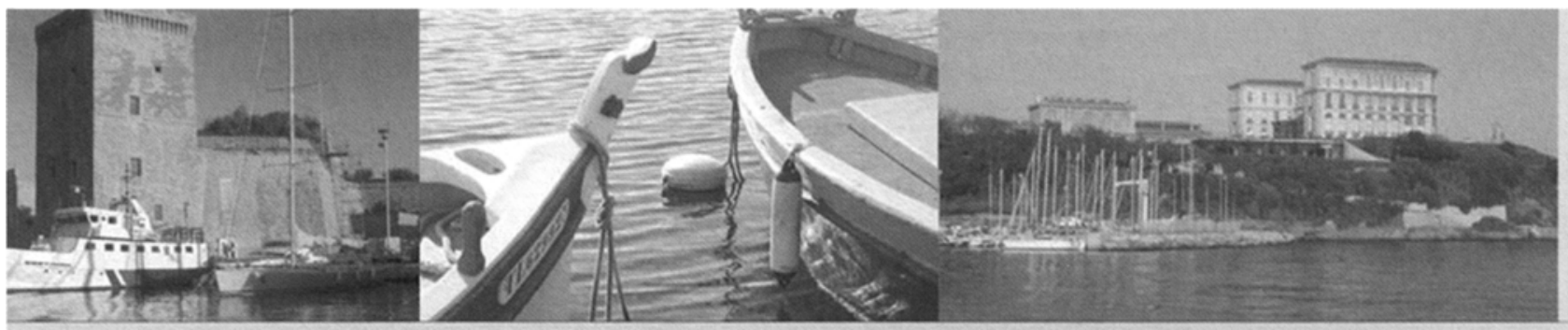

O LIEU DU CONGRÈS

Sofitel PaLm Beach

200 Corniche Kennedy 13007 Marseille

Tel : (+33)4/91161900 - Fax : (+33)4/91161939

Email : H3485@accor.com • Url : http://www.sofitel.com

Parking extérieur gratuit à proximité

\section{O DEJEUNERS}

Les déjeuners se prendront au Sofitel Palm Beach sous forme de buffet au centre du Hall d'exposition. Les déjeuners sont compris dans le droit d'inscription. Merci d'indiquer impérativement sur le bulletin d'inscription si vous souhaitez y participer.

\section{O CERTIFICATION FORMATION MEDICALE CONTINUE (FMC)}

Le congrès de la SALF est certifié FMC par l'Association Française d'Urologie (AFU). Le numéro de référence est le 163 et le coefficient C15 (catégorie-Nombre d'unité).

\section{O PUBLICATION DES COMMUNICATIONS}

Chaque communication fera l'objet d'une publication dans la revue ANDROLOGIE (Rédacteur en chef : Robert Mieusset)

\section{O PARTENARIAT DE LA SALF}

La SALF attribue des bourses, des prix DEA, DESS et des prix posters grâce au soutien du laboratoire Pfizer (partenaire depuis 1999).

\section{REDUCTION AIR FRANCE :}

\section{AIR FRANCE}

Ce document original vous permettra d'obtenir jusqu'à $45 \%$ de réduction sur le plein tarif d'un aller-retour en classe économique (soumis à conditions) sur le réseau France métropolitaine pour vous rendre à cette manifestation.

Réservation du billet électronique :

Pour réserver et obtenir votre billet électronique, contacter le $0820820820^{*}$ ou votre agence de voyage habituelle.

Homologation Réseau Métropole Air France AXZE SE 5510, Validité du 06/12 au 12/12/2005

- Sur le réseau France métropolitaine

Réductions enregistrées sur GGAIRAFCONGRES

Ce document est valable pour l'émission des billets nécessaires au déplacement de vos collaborateurs devant se rendre à la manifestation.

Un document nominatif sera exigé comme justificatif à tout moment du voyage (confirmation d'inscription fournie par l'organisateur).

- communication tarifée : 0,12€/min

Société Air France, S.A. au capital de 1.901.231.625 euros - RCS Bobigny 420495178 Siège social : 45 rue de Paris, F95704 Roissy Charles de Gaulle cedex, France 


\section{INFORMATIONS GÉNÉRALES}

\section{- Appel aux propositions de posters}

- Les propositions de poster doivent être envoyées exclusivement par mail sous forme de résumé au secrétariat scientifique :

Professeur Marie GUICHAOUA - E-mail : mguichaoua@ap-hm.fr

- Date limite d'envoi des posters : VENDREDI 28 OCTOBRE 2005

- Consignes de rédaction des résumés : format numérique $\mathrm{PC}, 1$ page $\mathrm{A4}$ maximum, Word, Police Times New Roman taille 12, marges $2,5 \mathrm{~cm}$. Sur le résumé, merci de souligner l'auteur qui présentera le poster

- Les posters seront affichés dans le Hall d'exposition pendant toute la durée du congrès

- Au cours du congrès, un jury sélectionnera les 6 meilleurs posters pour une présentation orale de 6 minutes. Les présentations seront suivies d'une remise des prix. Dans l'hypothèse d'une sélection, merci de préparer une présentation de 3 diapositives maximum, numérique, formatée PC, enregistrée sous power point sur support CD-Rom ou clé USB

- Un poster ne pourra être affiché et faire partie de la sélection que si son auteur est inscrit au congrès

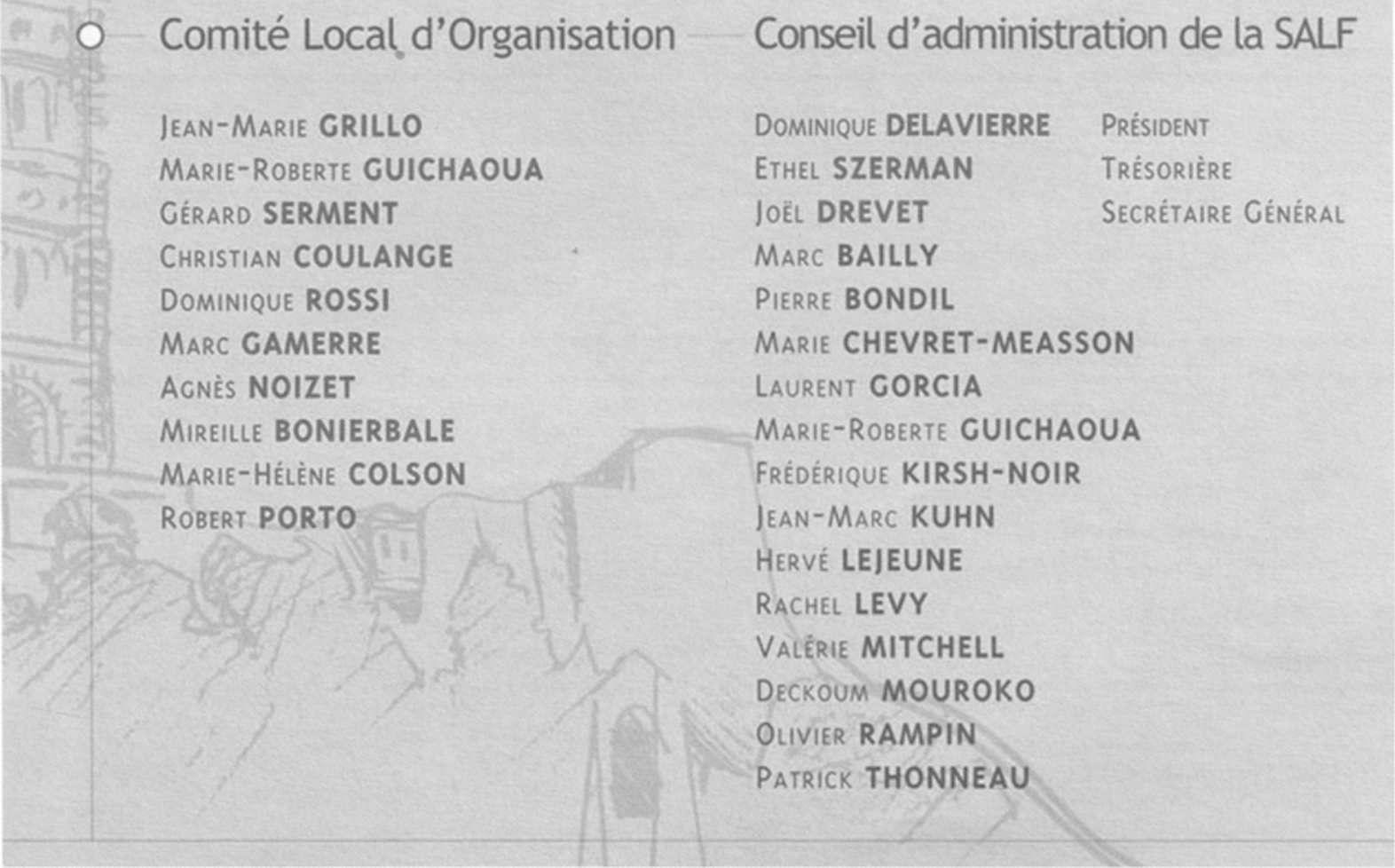

